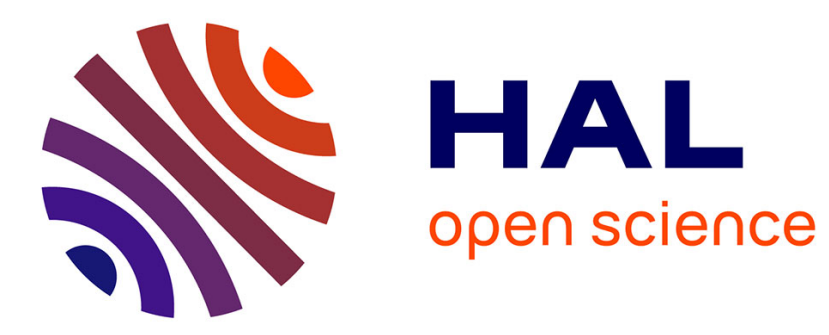

\title{
Immersion and Invariance Control for Lateral Dynamics of Autonomous Vehicles, with Experimental Validation
}

Reine Talj, Gilles Tagne, Ali Charara

\section{To cite this version:}

Reine Talj, Gilles Tagne, Ali Charara. Immersion and Invariance Control for Lateral Dynamics of Autonomous Vehicles, with Experimental Validation. European Control Conference (ECC 2013), Jul 2013, Zurich, Switzerland. pp.968-973. hal-00879626

\section{HAL Id: hal-00879626 https://hal.science/hal-00879626}

Submitted on 4 Nov 2013

HAL is a multi-disciplinary open access archive for the deposit and dissemination of scientific research documents, whether they are published or not. The documents may come from teaching and research institutions in France or abroad, or from public or private research centers.
L'archive ouverte pluridisciplinaire HAL, est destinée au dépôt et à la diffusion de documents scientifiques de niveau recherche, publiés ou non, émanant des établissements d'enseignement et de recherche français ou étrangers, des laboratoires publics ou privés. 


\title{
Immersion and Invariance Control for Lateral Dynamics of Autonomous Vehicles, with Experimental Validation
}

\author{
Reine Talj, Gilles Tagne and Ali Charara
}

\begin{abstract}
Autonomous intelligent vehicles are under intensive development, especially this last decade. The autonomous navigation consists on developing three main key steps: 1. environment perception, 2. path planning and decision, and 3. vehicle control. This paper focus on the lateral control of intelligent vehicles ; it presents design and experimental validation of a vehicle lateral controller based on Immersion and Invariance $(I \& I)$ principle, to minimize the lateral displacement of the autonomous vehicle with respect to a given reference trajectory. The control input is the steering angle and the output is the lateral error displacement. The closed-loop system simulated on Matlab-Simulink has been compared to the experimental data acquired on our vehicle DYNA, a Peugeot 308 , according to several driving scenarios. The experimental validation shows robustness and good performance of the proposed control approach.
\end{abstract}

\section{INTRODUCTION}

Technological advances in recent years have favored the emergence of intelligent vehicles with the capacity to anticipate and compensate a failure (of driver, vehicle or infrastructure) or even to ensure an autonomous driving.

An autonomous navigation requires three main steps: 1) the perception that consists on detecting the dynamical environment of the vehicle including road, fix and mobile obstacles ; 2) the path planning that consists to generate and choose one trajectory (reference path) in the navigable space, according to several criteria ; and 3) the vehicle control that consists to handle the vehicle using actuators like brake, accelerator and steering wheel to follow the reference path.

This paper focus on the third main step, that treat the vehicle control. And more precisely, the lateral control of the intelligent vehicle. This is a very active research field that has been studied since the 1950s Lateral control consists on automatically steering the vehicle to follow the reference trajectory. Given the high nonlinearity of the vehicle system on one hand, and the uncertainties and disturbances of such a system on the other hand, a very important issue to be considered in the control design is the robustness. The controller should be able to reject the disturbances caused by wind, coefficient of friction of the road and many other reasons, and able to deal with parameter uncertainties and variations.

For over 20 years, considerable research is conducted to provide lateral guidance of autonomous vehicles. Several

Reine Talj, Gilles Tagne and Ali Charara are working at Heudiasyc Laboratory, UMR CNRS 7253, Université de Technologie de Compiègne, BP 20529, 60205 Compiègne, France

reine.taljahds.utc.fr, gilles.tagne@hds.utc.fr, ali.charara@hds.utc. fr control strategies have been developed in the literature. Simple Proportional (P) and Proportional-Integral (PI) controllers have been proposed in [1]. Also a nested PID controller is presented in [2]. On the other hand, adaptive controllers have been developed for this application, as in [3]. Moreover, different other classical techniques have been applied: $H_{\infty}$ control in [4], state feedback control in [5], Lyapunov stability based control in [6], artificial intelligence in [7], fuzzy logic in [8], linear quadratic optimal predictive control in [9], and many other techniques.

Robust control as the sliding mode technique has been applied to the lateral control in [10] and [11]. This control strategy is well suited to driving conditions, given its robustness against uncertainties and its capacity to reject disturbances encountered in automotive applications. However, its main drawback is the chattering.

On the other hand, Model Predictive Control (MPC) appears to be well suited to the trajectory following [12], [13]. It allows to consider the problem of trajectory tracking for nonlinear systems taking into account the constraints on the state variables and/or control inputs. However, the computation time increases considerably at high speed autonomous driving, what renders difficult the use of such method in real-time operation [9].

With such advances in this domain, the proposed controllers have been subject of several performance comparison as in [14], where a comparison is made between proportional, adaptive, $H_{\infty}$ and fuzzy controllers. Different comparisons showed that the class of adaptive controllers represents a very promising technique for such uncertain and nonlinear application.

In this paper, we present a vehicle lateral controller based on Immersion and Invariance $(I \& I)$ principle. The $I \& I$ is a relatively new method for designing nonlinear and adaptive controllers for (uncertain) nonlinear systems [15], [16]. The method relies upon the notions of system immersion and manifold invariance. The basic idea of the $I \& I$ approach is to achieve the control objective by immersing the plant dynamics into a (possibly lower-order) target system that captures the desired behavior [15]. This is achieved by finding a manifold in state-space that can be rendered invariant and attractive -with internal dynamics that reflect the desired closed-loop dynamics- and by designing a control law that takes the state of the system towards the manifold [17]. The advantage is to reduce the controller design problem to other subproblems which might be easier to solve. To design the controller, we consider that the vehicle is equipped with all the necessary sensors to measure lateral acceleration, 
yaw rate and other variables. To validate the proposed approach, tests were made with real data acquired on our vehicle DYNA, on the tracks and circuits of CERAM ${ }^{1}$. The experimental results show the performance and robustness of the proposed approach.

This paper is organized as follows. Section II presents the dynamical models of the vehicle, used for control design and validation. In Section III, the $I \& I$ main principle is presented and the control strategy for lateral vehicle dynamics is developed. Section IV presents the experimental results and the performance validation of the proposed controller. Finally, we conclude in Section V, with some remarks and future work directions.

\section{VEHICLE LATERAL DYNAMICS MODEL AND CONTROL PROBLEM DEFINITION}

In this work, we use two vehicle models. The first one is the bicycle model used in Section III.B for the control design. The second is the 4-wheel vehicle model used to validate in simulation the proposed controller in closed loop. In the following we develop the bicycle model.

To represent the vehicle with a simple model suitable for control applications, a widely used dynamic bicycle model is considered [5].

This model is used to represent the lateral vehicle behavior (lateral acceleration, yaw rate, sideslip angle) and assumes that the vehicle is symmetrical, and sideslip angles on the same axle are equal. The roll and pitch dynamics are neglected and angles are assumed to be small (steering, sideslip, yaw).

With a linear tire force model we obtain a linear parameter varying model (LPV), the longitudinal velocity $V_{x}$ is considered as a varying parameter. Dynamic equations of the bicycle model are given by:

$$
\left\{\begin{array}{l}
\ddot{y}=-\frac{\left(C_{f}+C_{r}\right)}{m V_{x}} \dot{y}-\left(\frac{L_{f} C_{f}-L_{r} C_{r}}{m V_{x}}+V_{x}\right) \dot{\psi}+\frac{C_{f}}{m} \delta \\
\ddot{\psi}=-\frac{L_{f} C_{f}-L_{r} C_{r}}{I_{z} V_{x}} \dot{y}-\frac{L_{f}^{2} C_{f}+L_{r}^{2} C_{r}}{I_{z} V_{x}} \dot{\psi}+\frac{L_{f} C_{f}}{I_{z}} \delta
\end{array}\right.
$$

where $y$ and $\psi$ represent respectively the lateral position and the yaw angle of the vehicle. Table I presents vehicle nomenclature and parameters.

TABLE I

Vehicle Nomenclature ANd Parameters (BiCyCle Model)

\begin{tabular}{|c||c||c||c|}
\hline$V_{x}$ & Longitudinal velocity & - & {$[\mathrm{m} / \mathrm{s}]$} \\
\hline$\dot{y}$ & Lateral velocity & - & {$[\mathrm{m} / \mathrm{s}]$} \\
\hline$\dot{\psi}$ & Yaw rate & - & {$[\mathrm{rad} / \mathrm{s}]$} \\
\hline$\delta$ & steering wheel angle & - & {$[\mathrm{rad}]$} \\
\hline $\mathrm{m}$ & Mass & 1719 & {$[\mathrm{~kg}]$} \\
\hline$I_{z}$ & Yaw moment of inertia & 3300 & {$\left[\mathrm{kgm}^{2}\right]$} \\
\hline$L_{f}$ & Front axle-COG distance & 1.195 & {$[\mathrm{~m}]$} \\
\hline$L_{r}$ & Rear axle-COG distance & 1.513 & {$[\mathrm{~m}]$} \\
\hline$C_{f}$ & Cornering stiffness of the front tire & 170550 & {$[\mathrm{~N} / \mathrm{rad}]$} \\
\hline$C_{r}$ & Cornering stiffness of the rear tire & 137844 & {$[\mathrm{~N} / \mathrm{rad}]$} \\
\hline
\end{tabular}

${ }^{1}$ CERAM -"Centre d'Essais et de Recherche Automobile de Mortefontaine" is an automobile testing and research center located in France.
We consider now the error variables $e_{1}$ and $e_{2}$ defined as follows:

$$
\ddot{e}_{1}=\ddot{y}+V_{x}\left(\dot{\psi}-\dot{\psi}_{\text {des }}\right), \quad e_{2}=\psi-\psi_{\text {des }}
$$

where $e_{1}$ and $e_{2}$ represent respectively the lateral position error or displacement and the yaw angle error with respect to road.

The error dynamical equations are the following,

$$
\left\{\begin{array}{l}
\ddot{e}_{1}=-\frac{C_{0}}{m V_{x}} \dot{e}_{1}-\frac{C_{1}}{m V_{x}} \dot{e}_{2}+\frac{C_{0}}{m} e_{2}+\frac{C_{f}}{m} \delta-\left(\frac{C_{1}}{m V_{x}}+V_{x}\right) \dot{\psi}_{d e s} \\
\ddot{e}_{2}=-\frac{C_{1}}{I_{z} V_{x}} \dot{e}_{1}-\frac{C_{2}}{I_{z} V_{x}} \dot{e}_{2}+\frac{C_{1}}{I_{z}} e_{2}+\frac{L_{f} C_{f}}{I_{z}} \delta-\frac{C_{2}}{I_{z} V_{x}} \dot{\psi}_{d e s}
\end{array}\right.
$$

where $\dot{\psi}_{d e s}=\frac{V_{x}}{R}$, with $R$ the radius of curvature, and

$$
C_{0}=C_{f}+C_{r}, \quad C_{1}=C_{f} L_{f}-C_{r} L_{r}, \quad C_{2}=C_{f} L_{f}^{2}+C_{r} L_{r}^{2} .
$$

Hence, the dynamical model of the system can be written in the form $\dot{x}=f(x, u)$ with $x=\left[x_{1}, x_{2}, x_{3}, x_{4}\right]=\left[\dot{e}_{1}, e_{1}, \dot{e}_{2}, e_{2}\right]^{\top}$ and $u=[\delta, \rho], \rho=1 / R$ representing the curvature of the road. The dynamical equations are,

$$
\begin{aligned}
& \dot{x}_{1}=-\frac{C_{0}}{m V_{x}} x_{1}-\frac{C_{1}}{m V_{x}} x_{3}+\frac{C_{0}}{m} x_{4}+\frac{C_{f}}{m} \delta-\left(\frac{C_{1}}{m}+V_{x}^{2}\right) \rho, \\
& \dot{x}_{2}=x_{1}, \\
& \dot{x}_{3}=-\frac{C_{1}}{I_{z} V_{x}} x_{1}-\frac{C_{2}}{I_{z} V_{x}} x_{3}+\frac{C_{1}}{I_{z}} x_{4}+\frac{L_{f} C_{f}}{I_{z}} \delta-\frac{C_{2}}{I_{z}} \rho, \\
& \dot{x}_{4}=x_{3} .
\end{aligned}
$$

The control input $\delta$ has a direct influence on the dynamics of both state variables $x_{1}$ and $x_{3}$. To reduce the number of variables whose dynamics depend explicitly on the control input, we proceed to the following change of variable:

$$
\eta_{3}=x_{3}-\frac{m L_{f}}{I_{z}} x_{1}
$$

The dynamic equation of the new state variable $\eta_{3}$ can be written as follows,

$$
\begin{aligned}
\dot{\eta}_{3}= & F_{\eta_{3}} x_{1}+\frac{C_{1} L_{f}-C_{2}}{I_{z} V_{x}} \eta_{3}-\frac{C_{0} L_{f}-C_{1}}{I_{z}} x_{4} \\
& +\frac{L_{f} C_{1}-C_{2}+m L_{f} V_{x}^{2}}{I_{z}} \rho,
\end{aligned}
$$

with

$$
F_{\eta_{3}}=\frac{C_{0} L_{f}-C_{1}}{I_{z} V_{x}}+\frac{m L_{f}}{I_{z}}\left(\frac{C_{1} L_{f}-C_{2}}{I_{z} V_{x}}\right) .
$$

The aim of the lateral control is to cancel the lateral error displacement and the yaw rate error. Then, for a given curvature $\rho$ and longitudinal velocity $V_{x}$, the desired behavior corresponds to $x_{1}=x_{2}=x_{3}=0$. Hence, the desired equilibrium point is:

$$
\left(x_{1}, x_{2}, \eta_{3}, x_{4}\right)^{\top}=\left(0,0,0, x_{4}^{\star}\right)^{\top}
$$

with

$$
x_{4}^{\star}=\frac{L_{f} C_{1}-C_{2}+m L_{f} V_{x}^{2}}{C_{0} L_{f}-C_{1}} \rho .
$$


We define the new variable

$$
\eta_{4}=x_{4}-x_{4}^{\star} .
$$

Hence, the new state vector having the origin as a desired equilibrium point is $\eta=\left[\eta_{1}, \eta_{2}, \eta_{3}, \eta_{4}\right]^{\top}=$ $\left[x_{1}, x_{2},\left(x_{3}-\frac{m L_{f}}{I_{z}} x_{1}\right),\left(x_{4}-x_{4}^{\star}\right)\right]^{\top}$. The model dynamics be-
come,

$$
\begin{aligned}
\dot{\eta}_{1}= & -\left[\frac{C_{0}}{m V_{x}}+\frac{C_{1} L_{f}}{I_{z} V_{x}}\right] \eta_{1}-\frac{C_{1}}{m V_{x}} \eta_{3}+\frac{C_{0}}{m} \eta_{4}+\frac{C_{f}}{m} \delta \\
& +\frac{C_{0}}{m} x_{4}^{\star}-\left(\frac{C_{1}}{m}+V_{x}^{2}\right) \rho \\
\dot{\eta}_{2}= & \eta_{1}, \\
\dot{\eta}_{3}= & F_{\eta_{3}} \eta_{1}+\frac{C_{1} L_{f}-C_{2}}{I_{z} V_{x}} \eta_{3}-\frac{C_{0} L_{f}-C_{1}}{I_{z}} \eta_{4}, \\
\dot{\eta}_{4}= & \frac{m L_{f}}{I_{z}} \eta_{1}+\eta_{3} .
\end{aligned}
$$

In this system, it can be noticed that the control input $\delta$ representing the steering angle acts directly on the variable $\eta_{1}$, and acts implicitly on the other state variables dynamics via $\eta_{1}$.

Given that the lateral vehicle controller aims mainly to cancel the lateral error displacement with respect to a reference trajectory, then the target behavior of the system corresponds to $\dot{e}_{1}=e_{1}=0$, which is equivalent to $\eta_{1}=\eta_{2}=$ 0 .

To compare our simulation results with experimental data, we used a more representative 4-wheel model to represent the vehicle dynamics and Dugoff's tire model for longitudinal and lateral tire forces. In this model, longitudinal, lateral, yaw and roll dynamics are considered.

\section{IMMERSION AND INVARIANCE $(I \& I)$ CONTROLLER DESIGN}

\section{A. I\&I main principle}

The developed controller is based on the following theorem, representing the main stabilization result of the Immersion and Invariance method.

Theorem 1: [15] Consider the system

$$
\dot{x}=f(x)+g(x) u,
$$

with $x \in \mathbb{R}^{n}, u \in \mathbb{R}^{m}$, and an equilibrium point $x^{\star}$ to be stabilized. Assume that there exist smooth mappings $\alpha$ : $\mathbb{R}^{p} \rightarrow \mathbb{R}^{p}, \pi: \mathbb{R}^{p} \rightarrow \mathbb{R}^{n}, \phi: \mathbb{R}^{n} \rightarrow \mathbb{R}^{n-p}, c: \mathbb{R}^{p} \rightarrow \mathbb{R}^{m}$ and $v: \mathbb{R}^{n x(n-p)} \rightarrow \mathbb{R}^{m}$, with $p<n$, such that the following hold.

- (A1) The target system

$$
\dot{\xi}=\alpha(\xi),
$$

with $\xi \in \mathbb{R}^{p}$ has a globally asymptotically stable equilibrium at $\xi^{\star} \in \mathbb{R}^{p}$ and

$$
x^{\star}=\pi\left(\xi^{\star}\right) .
$$

- (A2) For all $\xi \in \mathbb{R}^{p}$,

$$
f(\pi(\xi))+g(\pi(\xi)) c(\pi(\xi)))=\frac{\partial \pi}{\partial \xi} \alpha(\xi) .
$$

- (A3) The set identity

$$
\left\{x \in \mathbb{R}^{n} \mid \phi(x)=0\right\}=\left\{x \in \mathbb{R}^{n} \mid x=\pi(\xi), \xi \in \mathbb{R}^{p}\right\}
$$

holds.

- (A4) All trajectories of the system

$$
\begin{aligned}
\dot{z} & =\frac{\partial \phi}{\partial x}(f(x)+g(x) v(x, z)), \\
\dot{x} & =f(x)+g(x) v(x, z),
\end{aligned}
$$

are bounded and (16) has a uniformly globally asymptotically stable equilibrium at $z=0$.

Then $x^{\star}$ is a globally asymptotically stable equilibrium of the closed-loop system

$$
\dot{x}=f(x)+g(x) v(x, \phi(x)) .
$$

Any trajectory $x(t)$ of the closed-loop system $\dot{x}=f(x)+g(x) v(x, \phi(x))$ is the image through the mapping $\pi($.$) of a trajectory \xi(t)$ of the target system. Note that the mapping $\pi: \xi \rightarrow x$ is an immersion, i.e., the rank of $\pi$ is equal to the dimension of $\xi$. Then, the approach consists on applying a control law that renders the manifold $x=\pi(\xi)$ attractive and keeps the closed-loop trajectories bounded.

\section{B. I\&I application on vehicle lateral control}

Consider the vehicle lateral dynamical model (10). As mentioned before, the main objective of the steering controller is to cancel the lateral error displacement with respect to a given trajectory, then $\eta_{1}=\eta_{2}=0$ at the equilibrium.

Hence, the target dynamical system $\left(\xi_{1}, \xi_{2}\right)$ has been chosen as the image of the subsystem $\left(\eta_{3}, \eta_{4}\right)$ when $\eta_{1}=$ $\eta_{2}=0$. The target dynamics can be expressed as follows,

$$
\begin{aligned}
& \dot{\xi}_{1}=\frac{C_{1} L_{f}-C_{2}}{I_{z} V_{x}} \xi_{1}-\frac{C_{0} L_{f}-C_{1}}{I_{z}} \xi_{2}, \\
& \dot{\xi}_{2}=\xi_{1} .
\end{aligned}
$$

Proposition 1: The target model (19) has a globally asymptotically stable equilibrium at the origin $(0,0)$.

Proof: If we neglect the curvature variation, the dynamics of the state vector $\xi=\left[\xi_{1}, \xi_{2}\right]$ can be written in the form

$$
\dot{\xi}=A \xi
$$

with

$$
A=\left(\begin{array}{cc}
\frac{C_{1} L_{f}-C_{2}}{I_{z} V_{x}} & -\frac{C_{0} L_{f}-C_{1}}{I_{z}} \\
1 & 0
\end{array}\right),
$$

Given the characteristics of the system, we have

$$
C_{1} L_{f}-C_{2}<0, \quad C_{0} L_{f}-C_{1}>0 .
$$

Then, after some simple calculations, one can prove that the matrix $A$ verifies the Routh-Hurwitz stability criterion, what yields to the desired result.

Proposition 2: Consider the system (10) of the form $\dot{\eta}=$ $f(\eta, \delta)$, and having the equilibrium point at the origin. Moreover, the system (19), which is the image of $\left(\eta_{3}, \eta_{4}\right)$ for $\eta_{1}=\eta_{2}=0$, has a globally asymptotically stable equilibrium 
at the origin. Then, the system (10) is (globally) I\&Istabilisable with target dynamics (19).

Proof: We define now the off-the-manifold variable

$$
z=\eta_{1}+\lambda \eta_{2}, \quad \text { s.t. } \lambda>0
$$

We have to select a control input $\delta$ such that the trajectories of the closed-loop system are bounded and $z=\eta_{1}+\lambda \eta_{2}$ converges to zero. Notice that,

$$
\text { If }(z=0) \Rightarrow \eta_{1}+\lambda \eta_{2}=0 \Rightarrow \dot{\eta}_{2}+\lambda \eta_{2}=0
$$

Hence, when $z \rightarrow 0, \eta_{2}$ converges exponentially to zero with the rate of convergence $\lambda$. Then, $\eta_{1}=\dot{\eta}_{2}$ converges also to zero, yielding to the desired result. To this end, let $\dot{z}=-K(\eta) z$ with $K(\eta)>0$ for any $\eta . K(\eta)$ represents the rate of exponential convergence of $z$ to zero.

Replacing $\dot{z}$ and $z$ by their expressions function of $\dot{\eta}_{1}, \dot{\eta}_{2}$, $\eta_{1}$ and $\eta_{2}$, we have $\dot{z}$ in the form

$\dot{z}=h(\eta)+b \delta=-K(\eta) z \quad \Rightarrow \quad \delta=-\frac{h(\eta)}{b}-\frac{K(\eta)}{b} z$.

and after some calculations, one can find that the corresponding control input has the following expression

$$
\begin{aligned}
\delta= & \frac{m}{C_{f}}\left\{-K(\eta)\left[\eta_{1}+\lambda \eta_{2}\right]+\left[\frac{C_{0}}{m V_{x}}+\frac{C_{1} L_{f}}{I_{z} V_{x}}-\lambda\right] \eta_{1}\right. \\
& \left.+\frac{C_{1}}{m V_{x}} \eta_{3}-\frac{C_{0}}{m} \eta_{4}-\frac{C_{0}}{m} x_{4}^{\star}+\left[\frac{C_{1}}{m}+V_{x}^{2}\right] \rho\right\} .
\end{aligned}
$$

Given the structure of the system, it can be noticed that there are two manifolds, an outer manifold $\mathscr{M}_{2}$ reached when the off-the-manifold variable $z$ converges exponentially to zero with the rate of convergence $K(\eta)>0$. Then, an inner manifold $\mathscr{M}_{1}$ can be reached from any point in $\mathscr{M}_{2}$ with the off-the-manifold variable $\eta_{2}$ that converges exponentially to zero with the rate of convergence $\lambda>0$. Finally the manifold $\mathscr{M}_{1}$ contains the target model having a globally asymptotically stable equilibrium at the origin. $K(\eta)$ and $\lambda$ have to be chosen in such a way to insure the incremental attractivity of both manifolds, then global asymptotic stability of the system can be assured.

To prove boundedness of $\xi=\left(\xi_{1}, \xi_{2}\right)$ in the manifold $\mathscr{M}_{1}$, pick any $M>0$ and let $V_{1}(\xi)$ be a positive-definite and proper function such that

$$
\nabla V_{1}^{\top} f(\xi)<0,
$$

for all $\|\xi\|>M$. Note that such a function $V_{1}(\xi)$ exists, by global asymptotic stability of the zero equilibrium of the system $\dot{\xi}=f(\xi)=A \xi$, but $V_{1}(\xi)$ is not necessarily a Lyapunov function for $\dot{\xi}=f(\xi)$.

The function $V_{1}(\xi)$ is chosen to be

$$
V_{1}(\xi)=\frac{1}{2} \xi_{1}^{2}+\frac{1}{2} \xi_{2}^{2} .
$$

$$
\begin{aligned}
\dot{V}_{1}(\xi) & =\xi_{1} \dot{\xi}_{1}+\xi_{2} \dot{\xi}_{2}, \\
& =\frac{C_{1} L_{f}-C_{2}}{I_{z} V_{x}} \xi_{1}^{2}-\left[\frac{C_{0} L_{f}-C_{1}}{I_{z}}-1\right] \xi_{1} \xi_{2}, \\
\dot{V}_{1}(\xi) & <0
\end{aligned}
$$

for all $\|\xi\|>M$. Note that $\xi_{1}$ and $\xi_{2}$ correspond to the variables $\eta_{3}$ and $\eta_{4}$ respectively when $\eta_{1}=\eta_{2}=0$. Consider now the positive definite and proper function $V_{2}\left(-\lambda \eta_{2}, \eta_{2}, \eta_{3}, \eta_{4}\right)$ defined in the manifold $\mathscr{M}_{2}$, that correspond to $z=\eta_{1}+\lambda \eta_{2}=0$.

$$
V_{2}(\eta, z=0)=\underbrace{\frac{1}{2} \eta_{3}^{2}+\frac{1}{2} \eta_{4}^{2}}_{V_{1}(\eta)}+\frac{1}{2} \eta_{2}^{2}
$$

Given that $\eta_{1}=\dot{\eta}_{2}=-\lambda \eta_{2}$ for $z=0$, then,

$$
\begin{aligned}
\dot{V}_{2}(\eta, z & =0)=\dot{V}_{1}(\xi)+F_{\eta_{3}} \eta_{1} \eta_{3}+\frac{m L_{f}}{I_{z}} \eta_{1} \eta_{4}+\eta_{2} \dot{\eta}_{2} \\
& =\dot{V}_{1}(\xi)-\left[F_{\eta_{3}} \eta_{3}+\frac{m L_{f}}{I_{z}} \eta_{4}\right] \lambda \eta_{2}-\lambda \eta_{2}^{2} \\
& \leq \dot{V}_{1}(\xi)+\frac{\left[F_{\eta_{3}} \eta_{3}+\frac{m L_{f}}{I_{z}} \eta_{4}\right]^{2}}{2 \gamma_{1}}+\frac{\gamma_{1}}{2} \lambda^{2} \eta_{2}^{2}-\lambda \eta_{2}^{2} \\
& \leq \underbrace{\dot{V}_{1}(\xi)+\underbrace{\left[F_{\eta_{3}} \eta_{3}+\frac{m L_{f}}{I_{z}} \eta_{4}\right]^{2}}}+\lambda \underbrace{\left(\frac{\gamma_{1}}{2} \lambda-1\right)} \eta_{2}^{2}
\end{aligned}
$$

for any $\gamma_{1}>0$. Setting $\gamma_{1}$ such that,

$$
\dot{V}_{1}(\xi)+\frac{\left[F_{\eta_{3}} \eta_{3}+\frac{m L_{f}}{I_{z}} \eta_{4}\right]^{2}}{2 \gamma_{1}}<0
$$

for all $\|\xi\|>M$, and setting $\lambda$ such that,

$$
\left(\frac{\gamma_{1}}{2} \lambda-1\right)<0 \Rightarrow \lambda<\frac{2}{\gamma_{1}}<-\frac{4 \dot{V}_{1}(\xi)}{\left[F_{\eta_{3}} \eta_{3}+\frac{m L_{f}}{I_{z}} \eta_{4}\right]^{2}}
$$

yield boundedness of trajectories in the manifold $\mathscr{M}_{2}$, and prove global asymptotic stability of the subsystem corresponding to $z=0$.

To complete the result, we define the positive definite and proper function $W(\eta)$ for the system (10) as follows,

$$
W(\eta)=\underbrace{\frac{1}{2} \eta_{3}^{2}+\frac{1}{2} \eta_{4}^{2}+\frac{1}{2} \eta_{2}^{2}}_{V_{2}(\eta)}+\frac{1}{2} z^{2} .
$$

Given that $\eta_{1}=\dot{\eta}_{2}=z-\lambda \eta_{2}$ in this system, then,

$$
\begin{gathered}
\dot{W}(\eta)=\dot{V}_{2}(\eta, z=0)+\left[F_{\eta_{3}} \eta_{3}+\frac{m L_{f}}{I_{z}} \eta_{4}\right] z+z \eta_{2}+z \dot{z}, \\
\leq \dot{V}_{2}(\eta, z=0)+\frac{\left[F_{\eta_{3}} \eta_{3}+\frac{m L_{f}}{I_{z}} \eta_{4}+\eta_{2}\right]^{2}}{2 \gamma_{2}}+\frac{\gamma_{2}}{2} z^{2}-K(\eta) z^{2}, \\
\leq \underbrace{\dot{V}_{2}(\eta, z=0)+\underbrace{\left[F_{\eta_{3}} \eta_{3}+\frac{m L_{f}}{I_{z}} \eta_{4}+\eta_{2}\right]^{2}}}+\underbrace{\left(\frac{\gamma_{2}}{2}-K(\eta)\right)} z^{2} .
\end{gathered}
$$


for any $\gamma_{2}>0$. Setting $\gamma_{2}$ such that,

$$
\dot{V}_{2}(\eta, z=0)+\frac{\left[F_{\eta_{3}} \eta_{3}+\frac{m L_{f}}{I_{z}} \eta_{4}+\eta_{2}\right]^{2}}{2 \gamma_{2}}<0,
$$

and setting $K(\eta)$ such that,

$$
\begin{aligned}
& \left(\frac{\gamma_{2}}{2}-K(\eta)\right)<0 \Rightarrow \\
& K(\eta)>\frac{\gamma_{2}}{2}>-\frac{\left[F_{\eta_{3}} \eta_{3}+\frac{m L_{f}}{I_{z}} \eta_{4}+\eta_{2}\right]^{2}}{4 \dot{V}_{2}(\eta, z=0)},
\end{aligned}
$$

yield the desired result of attractivity of the manifold $\mathscr{M}_{2}$ and global asymptotic stability of the closed-loop system with the $I \& I$ controller when the controller gains are chosen to verify the conditions (30) and (34).

\section{EXPERIMENTAL VALIDATION}

The experimental data used here are acquired on the CERAM test circuits by our vehicle DYNA. This vehicle is equipped with several sensors: an inertial measuring accelerations for $(\mathrm{x}, \mathrm{y}, \mathrm{z})$ and the yaw rate. The CORREVIT for measuring the sideslip angle and longitudinal velocity. Torque hubs for measuring tire-ground efforts and vertical loads on each tire. Four laser sensors to measure the height of the chassis. GPS and a CCD camera. Data provided via the CAN bus of the vehicle are also used, as the steering angle, the speed of rotation of the wheels.

To validate our control law, we perform several tests with our vehicle DYNA, according to different driving scenarios. The collected data are considered as reference data to be compared to the results obtained with the simulated closedloop system on Matlab-Simulink, with the proposed I\&I controller used to follow the reference trajectory given by the experimental data. Simulations were carried out with vehicle 4-wheel full model. For the control law, we used the nominal vehicle parameters (see Table 1) and the gains $k=10$ and $\lambda=8$. The robustness of the controlled system is tested with respect to speed and curvature variations, uncertainties and disturbances encountered in automotive applications. Several tests have been done during normal driving conditions, and showed that the controlled vehicle is able to track the reference path with small error.

\section{A. Robustness of the controller to strong nonlinear dynamics}

The test presented in Figures 1-(a), 1-(b) and 1-(c) consists on gradually increasing the speed while executing almost the same curvature (we fixed the radius of curvature around $50 \mathrm{~m}$ ). In this case, the lateral acceleration was significantly increasing and the vehicle's behavior becomes highly nonlinear. This type of test is used to assess the stability and robustness of the control law to strong nonlinear dynamics. This test also evaluates the effect of changing the vehicle speed.

Fig. 1-(b) shows that even when the longitudinal speed is fastly increasing (with a rate about $1 \mathrm{~m} / \mathrm{s}^{2}$ ) and the lateral acceleration reaches $8.5 \mathrm{~m} / \mathrm{s}^{2}$, the lateral displacement error remains small. In Fig. 1-(c) dynamic variables are very close to measured ones, even at high lateral acceleration up to $8 m / s^{2}$.

This test shows that the control law can ensure good performance even at the limit of stability when the lateral acceleration grows up to $8 \mathrm{~m} / \mathrm{s}^{2}$.

\section{B. Robustness of the controller to abrupt manœvers}

The second test presented in Figures 2-(a), 2-(b), and 2(c) executes an abrupt deceleration, reaching a low longitudinal speed less than $7 \mathrm{~m} / \mathrm{s}$. We noticed a relatively good performance with a lateral error displacement that does not exceed $6 \mathrm{~cm}$. But, in this experiment, we noticed also that in this test conditions of fast speed variation, a steady state error occurs in the closed-loop system. This is due to the controller structure. Indeed, from Equation (23) it can be noticed that the control input $\delta$ is composed of two terms: a term that compensates exactly $h(\eta)$ depending on the system variables, and based on the bicycle model, and another term $(-k(\eta) z)$ that insures the exponential convergence of $z$ to zero.

Given that the bicycle model is restricted to linear tire forces zone and to slowly varying longitudinal speed, when a nonlinear or abrupt manœuver occurs, a difference appears between the bicycle model and the 4-wheel representative model.

Hence, for strong nonlinear dynamics situations, the control input $\delta$ which is calculated with the bicycle model and applied on the real system is unable to ensure exact compensation of the term $h(\eta)$, then the variable $z$ converges to a $z^{\star} \neq 0$ in the neighborhood of zero. To remedy this problem, a robustness term $\delta_{r o b}$ is added to the control input, of the form

$$
\delta_{r o b}=-\beta \frac{|z|}{|z|+\varepsilon} \int \operatorname{sign}(z)
$$

that ensures a smooth convergence of $z$ to zero when the system is in the neighborhood of the origin. We take $\beta=$ 0.002 and $\varepsilon=0.01$. Figures 2-(b) and 2-(c) show the results of the closed-loop system, with and without the robustness additive term. It can be noticed in Fig. 2-(b) that the additive term $\delta_{r o b}$ canceled the lateral error displacement.

\section{CONCLUSIONS}

In this paper, a lateral dynamics controller for autonomous vehicles has been proposed. This control strategy is based on the Immersion and Invariance theory to provide robust lateral tracking of a reference trajectory at high speed. An experimental validation has been done according to several scenarios representing different driving situations. The performance and robustness of the controlled system have been tested with respect to speed and curvature variations. The performed tests highlighted the robustness of the developed control law. Finally, a robust term has been added to the controller to ensure exact path tracking even in high nonlinear situations at the limit of stability.

Given the implicit resemblance between the $I \& I$ and sliding mode principles, a comparison of the proposed 

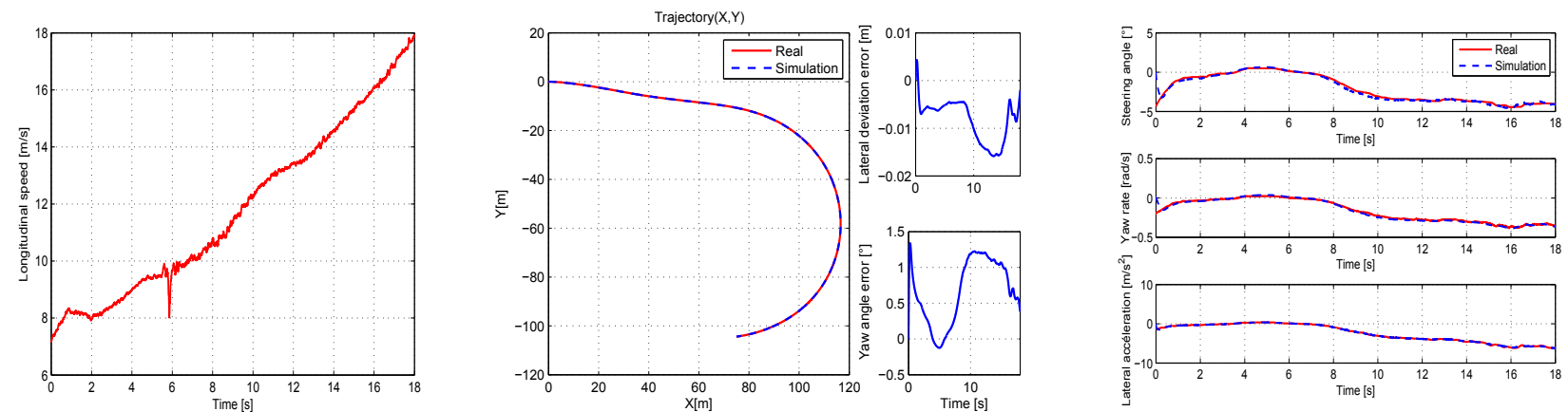

Fig. 1. Test 1: (a) Longitudinal speed, (b) Trajectory: Real (reference) and simulation (control law), (c) Steering angle, yaw rate and Lateral acceleration
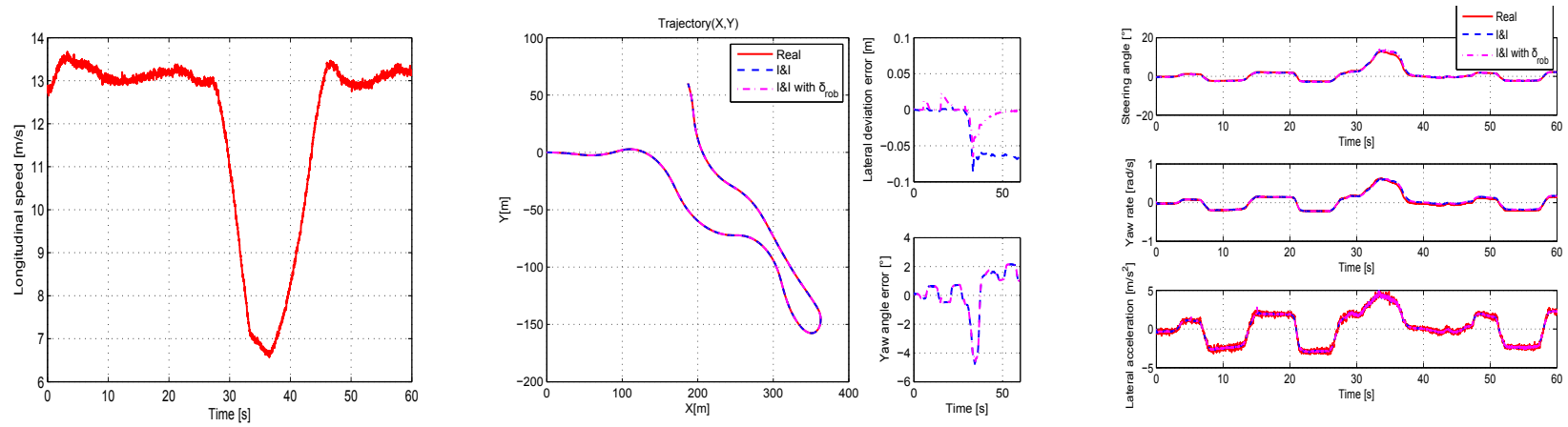

Fig. 2. Test 2: (a) Longitudinal speed, (b) Trajectory: Real (reference) and simulation (control law), (c) Steering angle, yaw rate and Lateral acceleration

control law with a higher order sliding mode control super-twisting algorithm - is under study. Note that the $I \& I$ reformulation of the stabilization problem is implicit in sliding mode control, where the target dynamics are the dynamics of the system on the sliding manifold, which is made attractive by a discontinuous control law. The main distinction between both control laws is that in $I \& I$, it is not necessarily required that the manifold be reached, however, in sliding mode the manifold must be reached in finite time.

In future work, the control law design will be extended to take into account the cant and the slope of the road. Moreover, a robotized vehicle arriving soon in the laboratory Heudiasyc, will allow us to test directly the control law on a robotized semi-autonomous vehicle.

\section{REFERENCES}

[1] A. Broggi, M. Bertozzi, and A. Fascioli, "The ARGO autonomous vehicle's vision and control systems," International Journal of Intelligent Control and Systems, vol. 3, no. 4, pp. 409-441, 1999.

[2] R. Marino, S. Scalzi, and M. Netto, "Nested PID steering control for lane keeping in autonomous vehicles," Control Engineering Practice, vol. 19, pp. 1459-1467, Dec. 2011.

[3] M. Netto, S. Chaib, and S. Mammar, "Lateral adaptive control for vehicle lane keeping," in American Control Conference, vol. 3, pp. 2693-2698, 2004.

[4] S. Hima, B. Lusseti, B. Vanholme, S. Glaser, and S. Mammar, "Trajectory Tracking for Highly Automated Passenger Vehicles," in IFAC World Congress, pp. 12958-12963, Aug. 2011.

[5] R. Rajamani, Vehicle dynamics and control. New York, USA: Springer, mechanical ed., 2006.

[6] A. Benine-Neto, S. Scalzi, S. Mammar, and M. Netto, "Dynamic controller for lane keeping and obstacle avoidance assistance system," in 13th International IEEE Conference on Intelligent Transportation Systems, pp. 1363-1368, Sept. 2010.
[7] E. Onieva, J. Naranjo, V. Milanés, J. Alonso, R. García, and J. Pérez, "Automatic lateral control for unmanned vehicles via genetic algorithms," Applied Soft Computing, vol. 11, pp. 1303-1309, Jan. 2011.

[8] J. Naranjo, C. Gonzalez, R. Garcia, and T. de Pedro, "Lane-Change Fuzzy Control in Autonomous Vehicles for the Overtaking Maneuver," IEEE Transactions on Intelligent Transportation Systems, vol. 9, pp. 438-450, Sept. 2008.

[9] D. Kim, J. Kang, and K. Yi, "Control strategy for high-speed autonomous driving in structured road," in 2011 14th International IEEE Conference on Intelligent Transportation Systems (ITSC), pp. 186-191, Oct. 2011.

[10] J. Ackermann, J. Guldner, W. Sienel, R. Steinhauser, and V. Utkin, "Linear and nonlinear controller design for robust automatic steering," IEEE Transactions on Control Systems Technology, vol. 3, pp. 132143, Mar. 1995.

[11] P. Hingwe and M. Tomizuka, "Experimental evaluation of a chatter free sliding mode control for lateral control in AHS," in Proceedings of the 1997 American Control Conference (Cat. No.97CH36041), vol. 5, pp. 3365-3369, 1997.

[12] P. Falcone, F. Borrelli, H. E. Tseng, J. Asgari, and D. Hrovat, "Linear time-varying model predictive control and its application to active steering systems: Stability analysis and experimental validation," Int. J. Robust Nonlinear Control, vol. 18, pp. 862-875, 2008.

[13] T. Besselmann and M. Morari, "Autonomous Vehicle Steering Using Explicit LPV-MPC,' in European Control Conference, (Budapest, Hungary), pp. 2628-2633, 2009.

[14] S. Chaib, M. Netto, and S. Mammar, "H /sub / adaptive, PID and fuzzy control: a comparison of controllers for vehicle lane keeping," in IEEE Intelligent Vehicles Symposium, no. 1, pp. 139-144, 2004.

[15] A. Astolfi, D. Karagiannis, and R. Ortega, Nonlinear and adaptive control with applications. Springer, 2008.

[16] A. Astolfi and R. Ortega, "Immersion and invariance: a new tool for stabilization and adaptive control of nonlinear systems," Automatic Control, IEEE Transactions on, vol. 48, no. 4, pp. 590-606, 2003.

[17] I. Sarras, H. Siguerdidjane, and R. Ortega, "An experimental result on stabilization via immersion and invariance : the cart-pendulum system," in Proceedings of the European Control Conference (ECC), (Budapest, Hungary), pp. 2152-2157, 23-26 August 2009. 